\title{
Automated Human Facial Expression and Emotion Detection: A Review
}

\author{
Akanksha Manuj \\ Computer Science \\ Department, \\ Student. \\ NMIMS University, India.
}

\author{
Supriya Agrawal \\ Computer Science \\ Department, \\ Assistant Professor \\ NMIMS University, India.
}

\begin{abstract}
Facial expression identifies the basic human emotions. It helps to tell the person by watching the image whether the person is actually telling the truth about what he is claiming or not. Human emotions detection is used in human computer interaction, military, law-enforcements, for autistic people and robotics etc. No one thought that detecting human emotions would be possible for a machine but computer science changed all that. Various techniques like Bezier curve, longest binary pattern and various classifiers have been implemented till now in this field. This paper will review the research work carried out in this field of human emotion detection from still images.
\end{abstract}

\section{Keywords}

Keywords-Emotions, Bezier Curve, Classifier, Facial Expression

\section{INTRODUCTION}

According to Dr. Charles Darwin, the facial expression indeed contributes in communicating one's emotions, opinions as well as intentions to each other in an effective way as stated in the paper [1]. Human emotions comprise of happiness, sad, anger, fear, disgust etc. It's used in a lot of fields like man machine,communication,security,law-enforcement,psychiatry, education, and telecommunications. It helps greatly in people affected by autism, whose emotions cannot be detected by humans naturally easily. A lot of companies are investing in it as it helps them better survey their products based on people's reactions and increase their profit. It doesn't deal with the rest of the body but only the facial features. The emotion detection process basically starts with segmentation, facial extraction and the detection of lips, eyes and the distance between the eyebrows etc. There are many methods which have been established for emotion detection but not all give an accurate result or are easy to implement .Each research till now in this field uses these steps according to the emotion needed for them to detect. The paper is a review of various recognition techniques and there implementation.

\section{ARCHITECTURE OF HUMAN EMOTION RECIGNITION SYSTEM}

In 1984, Wiliam James gave a very good psychological theory of human detection that first we see the thing and then react [5]. This reaction is known as human emotions that can be characterized in various ways like happy, sad, fear, surprise, anger and disgust. There are various building blocks for recognizing human emotion system as given below

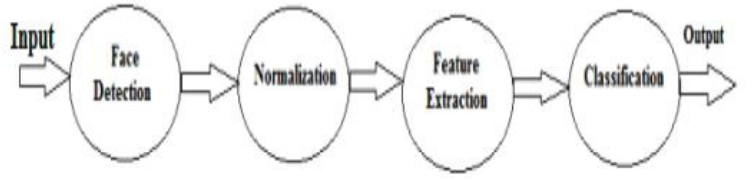

Fig 1: System Architecture of Facial Expression and Emotion Detection

\section{RELATED WORK TO RECOGNIZE}

This paper presents various methods involved in past to identify facial expression and emotion from still images. This paper mainly focuses on automated techniques for (i) Face Detection (ii) Feature Extraction (iii) Emotion Detection

\subsection{Skin Color Segmentation}

The first step after acquiring the image is segmenting it. Skin color segmentation is done in the paper [1] to separate the non-skin pixels from the skin pixels .Before applying this, the image is contrasted as a pre -requisite and for better results. This helps us getting closer to the next step of face detection .It eliminates the region not required, if suppose the person in the image is wearing a shirt then the shirt won't be seen in the output, only the face and the neck in some cases [1].

\subsection{Principle Component Analysis for Face Detection}

The face is where all the answers lie to the emotion expressed by a person so it's necessary that the algorithm chosen for the face is as accurate as possible or else it will be harder to detect the lips and the eyes. Principal Component Analysis is used to reduce the dimensionality of image and provides effective face indexing and retrieval. It is also known as the Eigen face approach [5]

\subsection{Bezier Curve and difference measurements Technique for Eyes and Lip detection}

Bezier curve and difference measurement is one the techniques to recognize human emotions using mathematical expressions [4]. Bezier curve is used to generate contour points considering global shape information with curve passing through first and last curve points [4].

\subsection{Emotion Detection using Pattern Matching}

Design the database which contains the values (patterns) of all types of emotions. Once all feature extraction is done, convert the obtained values in to large area and compare with already existing database. If pattern matches with happy emotion value then result will display as Happy Mood.[1] 


\section{PROBLEMS AND CHALLENGES}

Not much over, here was talked about the method of segmentation to be used but leaves it on the user[2] or doesn't explain the fact why it's better to choose skin color over binary [1].The paper in the first step does skin color segmentation but then goes back to binary for lip detection and eye detection. It becomes a hassle to go from one form to another for every step rather than having a binary throughout [1]. The methods mentioned in the paper can be strenuous and take time and may not be that accurate as it all depends on the symmetry of the face or the angle the picture is taken at, which may not be assumed in the paper [1]. The preprocessing methods are not much stressed on in the paper [2] leaving it to the user to decide how to deal with it. Though Eigen face helps achieving recognition of the human emotions in good amount of time and is helpful when having large amount of pictures in the database. It doesn't cope well with recognizing the change in expression. Bezier curve equation applies on facial feature to find middle point of two nearby objects. Bezier curve will not give correct results as every person has a different shape of lips [4].

Neural network is very appropriate and efficient when used to detect the face of a single person and when in a group photo or multiple people. K-clustering method is very good and should be used as a skin color segmentation method. The use of classifiers is intelligent .It reduces the much needed effort of double checking [2].

Human emotion can be detected easily using facial expression but it is little bit difficult to indentify using computer interface. This challenge motivates researchers to make an automated system to recognize human emotions in a very effective and reliable manner. An ideal expression detection system should identify the emotion detection of any age and gender. Such a system should also be invariant to different distraction like glasses, different hair styles, mustache, facial hairs and different lightening conditions [5]. Such type of system should detect correct facial expression analysis regardless of large changes in viewing condition and rigid movement [5]. Achieving optimal feature extraction and classification is a key challenge in this field because we have a huge variability in the input images. For better recognition result, most facial expressions recognition methods require more work to control imaging conditions like position and orientation of the face with respect to the camera.

\section{CONCLUSION}

In this paper various challenges and methods related to emotion detection are reviewed. These techniques focus on many factors namely face detection, lip detection, eye detection and various emotions detection. These recognition systems provide best result for some sets of data. The recognition rate was greater even with the small number of training images which demonstrated that it is fast, relatively simple, and works well in a constrained environment [3]. Though reviewed methods give $90-95 \%$ result and due to this, research in this field is still being carried out intensively. In future we will to come up with a system for easier detection of human emotions using better segmentation methods and wide range of classifiers like neural network ,support vector machines etc.: to simplify the process and give results as accurately as possible.

\section{REFERENCES}

[1] P. M. Chavan, Manan C Jadhav, Jinal B. Mashruwala, Aditi K. Nehete and Pooja A. Panjari .2013. Real Time Emotion Recognition through Facial Expressions for Desktop Devices (IJESE) India ISSN: 2319-6378, Vol 1 , Issue 7.

[2] Dilbag Singh.2012.Human Emotion Recognition System . I.J. Image, Graphics and Signal Processing, published by Modern Education and Computer Science, India .

[3] Henry A. Rowley, Shumeet Baluja, Takeo Kanade.1998.Neural Network-Based Face Detection.Appears in Computer Vision and Pattern Recognition.

[4] Pinky Rai, Manish Dixit.2013.Smile Detection via Bezier Curve of Mouth Interest Points. International Journal of Advance Research in Computer Science and Software Engineering, ISSN: 2277 128X Volume 3, Issue 7.

[5] M. Pantic, L.J.M. Rothkrantz.2004.Facial action recognition for facial expression analysis from static face images. IEEE Transactions on Systems, Man, and Cybernetics 34, (2004) 1449-1461M. Young

[6] Jyoti Rani,Kanwal garg.2014.Emotion detection Using Facial expressions-A review", International Journal of Advance Research in Computer Science and Software Engineering,ISSN:2277 128X Volume 4,Issue 4.

[7] Moon Hwan kim,Young Hoon Joo, Jim Bae Park.2005.Emotion Detection Algorithm Using Frontal Image.ICCAS,June(2-5),KINTEX,Gyeonggi -Do,Korea.

[8] Zhihong Zeng,Yun Fu,Glenn I.Roisman,Zhen Wen,Yuxiao Hu,Thomas S.Huang.2006.Spontaneous Emotional Facial Expression Detection.Journal of Multimedia,Vol 1,No.5,August 2006.

[9] Agata Kołakowska, Agnieszka Landowska, Mariusz Szwoch, Wioleta Szwoch, Michał R. Wróbel Gdansk .2013.Emotion Recognition and its application in software engineering.IEEE Explore Digital Library. 